CASE REPORT

\title{
Myelofibrosis and angiodysplasia of the colon: another manifestation of portal hypertension and massive splenomegaly?
}

\author{
T Biss, P Hamilton
}

J Clin Pathol 2004;57:999-1000. doi: 10.1136/icp.2003.015479

Bleeding owing to portal hypertensive colopathy, a form of large bowel angiodysplasia, as a cause of increased blood transfusion requirement is described in a 74 year old man with idiopathic myelofibrosis. The proposed mechanism and the potential therapeutic options for this rare complication of myelofibrosis are discussed.

A giodysplasia of the gastrointestinal tract causing blood loss and increased blood transfusion requirements in two patients with myelofibrosis was first described in two papers in 1991. ${ }^{12}$ In this report we describe what we believe to be only the third example of such an association, and one that we suggest is a consequence of portal hypertension, a recognised complication of myelofibrosis and associated gross splenomegaly.

"We suggest that this is an example of portal hypertensive colopathy, a manifestation of portal hypertension, which is a well recognised complication of patients with cirrhotic portal hypertension"

Although angiodysplasia of the lower gastrointestinal tract and the right side of the colon in particular is a well known cause of rectal bleeding in elderly patients, ${ }^{3}$ we illustrate here what our endoscopist reported as an unusual appearance for straightforward angiodysplasia. We suggest that this is an example of portal hypertensive colopathy, a manifestation of portal hypertension, which is a well recognised complication of patients with cirrhotic portal hypertension, also first reported in $1991 .^{4}$

We suspect that portal hypertensive colopathy may be an under recognised cause of gastrointestinal bleeding and increased blood transfusion requirement in patients with myelofibrosis with grossly enlarged spleens.

\section{CASE REPORT}

A 74 year old man presented with a one year history of progressive exertional breathlessness, leg cramps, and numbness of his feet. He had had several months of severe night sweats, poor appetite, and a weight loss of $7 \mathrm{~kg}$. Examination revealed $3 \mathrm{~cm}$ hepatomegaly and splenomegaly $9 \mathrm{~cm}$ below the costal margin. Full blood count revealed a haemoglobin of $80 \mathrm{~g}$ /litre, mean red cell volume of $79 \mathrm{fl}$, white blood cell count of $4.5 \times 10^{9} /$ litre, and platelets of $222 \times 10^{9}$ /litre. The blood film was leucoerythroblastic with anisopoikilocytosis and numerous tear drop poikilocytes seen.

The diagnosis derived from the clinical presentation and blood film morphology was idiopathic myelofibrosis, which was confirmed later by bone marrow examination when the trephine was almost completely replaced by fibrotic tissue. Residual areas of haemopoiesis showed hypercellularity, with increased numbers of megakaryocytes, with clustering and fibrotic entrapment.

He was begun on a blood transfusion programme, and obtained some symptomatic relief with a monthly transfusion of three units of blood. However, over the following months he began to complain of early satiety and continued weight loss, which was presumed to result from gastric compression caused by splenic enlargement, with intermittent left upper quadrant pain, often associated with left shoulder tip pain, thought be the result of recurrent splenic infarcts.

In an attempt to reduce the spleen size, transfusion requirement, and frequency of splenic infarcts, he was treated initially with hydroxyurea and dexamethasone, and later with stanozolol. These did not improve his symptoms or splenic size. His transfusion requirement increased gradually, rising to four units each month at one year from presentation, by which time he had developed massive hepatosplenomegaly.

At 22 months after presentation his transfusion requirement increased suddenly to four units of blood fortnightly. His spleen size was unchanged, confirmed by abdominal ultrasonography at $23 \mathrm{~cm}$ in length. Neither portal vein size/ flow nor the presence of ascites was commented on. Testing for faecal occult blood was complicated by the presence of haemorrhoids with intermittent small volume bleeding. An oesophago-gastroduodenoscopy showed extensive oesophageal candidiasis but no bleeding lesion or varices. He declined lower bowel investigation. After discharge he continued to require three units of blood fortnightly.

By 30 months, he was admitted as an emergency with left lower lobe pneumonia, which resolved with antimicrobials. During his recovery he had five episodes of fresh blood passed per rectum in one 24 hour period. It was then that he commented on the passage of fresh blood, either coating or mixed with his stool, on several occasions over the months before this admission. His platelet count at the time of bleeding was $52 \times 10^{9}$ /litre and his clotting screen was normal (prothrombin time, 14 seconds; activated partial thromboplastin time, 33 seconds; fibrinogen, $4.0 \mathrm{~g} / \mathrm{litre}$; and $\mathrm{D}$ dimer, $0.38 \mathrm{mg} /$ litre). Plain abdominal $x$ ray showed slightly dilated small bowel loops and thickening of the large bowel mucosa. He underwent emergency colonoscopy, which showed a very unusual appearance, with extensive telangiectatic submucosal vessels increasing with distance from the rectum to the caecum (fig 1). He was treated supportively with blood transfusion and the acute bleeding settled.

His recovery was complicated by post-pneumonic pleural effusion. Unfortunately, two months after discharge, some three and a half years after presentation, he had a further chest infection and died at home. 


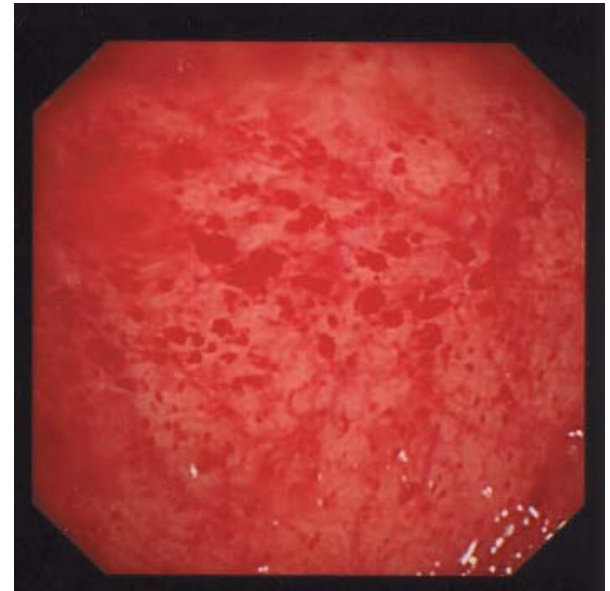

Figure 1 Endoscopic image of the right colon showing extensive friable telangiectatic lesions of the colonic mucosa.

\section{DISCUSSION}

Bleeding from the gastrointestinal tract in patients with myelofibrosis can be associated with oesophageal varices secondary to portal hypertension, which is a complication in some patients who have massive splenomegaly. ${ }^{5}$

Although no oesophageal varices were demonstrated in our patient, he did have haemorrhoids, which can be secondary to portal hypertension. In addition, extensive telangiectatic lesions were found throughout his colon. Telangiectatic lesions of the colon, termed portal hypertensive colopathy, are well recognised in patients with cirrhotic portal hypertension, ${ }^{4}$ and we suggest that portal hypertension is the mechanism for their occurrence in this case.

"This finding emphasises the importance of imaging the colon in addition to the upper gastrointestinal tract when searching for a cause of increased transfusion requirement in patients with myelofibrosis"

In 1991, Edoute et al reported a case of bleeding jejunoileal angiodysplasia in a patient with myelofibrosis resulting in recurrent malaena. ${ }^{1}$ Diagnosis was made by intraoperative endoscopy, following transfusion of a total of 65 units of blood to keep the haemoglobin value stable. Attempts were made to secure haemostasis by suturing of the angiodysplastic lesions, but the patient later died from massive gastrointestinal bleeding.

Freedman et al later reported the presence of bleeding telangiectatic lesions in the stomach, duodenum, and entire colon in a patient with myelofibrosis and massive hepatosplenomegaly. ${ }^{2}$ In this case, myelofibrosis had developed as a result of systemic mast cell disease. The telangiectatic lesions resolved completely within seven months of splenectomy, but the patient died later, cause unknown.

Our report of a third case confirms the association between angiodysplasia, which we attribute to portal hypertensive colopathy, and idiopathic myelofibrosis. This finding emphasises the importance of imaging the colon in addition to the
Take home messages

- We report the case of a 74 year old man with idiopathic myelofibrosis in whom bleeding as a result of portal hypertensive colopathy, a form of large bowel angiodysplasia, was the cause of increased blood transfusion requirement

- We believe that this case is only the third example of such an association, one that we suggest is a consequence of portal hypertension, a recognised complication of myelofibrosis and associated gross splenomegaly

- Portal hypertensive colopathy may be an under recognised cause of gastrointestinal bleeding and increased blood transfusion requirement in patients with myelofibrosis with grossly enlarged spleens

upper gastrointestinal tract when searching for a cause of increased transfusion requirement in patients with myelofibrosis.

There is limited evidence for effective treatment. Lesions may be too extensive to consider endoscopic cautery, laser treatment, or angiographic embolisation. Splenectomy may limit the development of further lesions but may lead to progressive hepatomegaly. Transjugular intrahepatic portosystemic shunt, oral propanolol, octreotide, and other measures to reduce portal pressure have been shown to be effective in some cases of portal hypertensive colopathy. ${ }^{6} 7$ Whether thalidomide with its anti-angiogenic properties may have a role is necessarily a speculative thought.

\section{Authors' affiliations}

T Biss, P Hamilton, Department of Haematology, Royal Victoria Infirmary, Newcastle upon Tyne NE1 4LP, UK

Correspondence to: Dr T Biss, Department of Haematology, Royal Victoria Infirmary, Queen Victoria Road, Newcastle upon Tyne NE1 4LP, UK; tbiss@dsl.pipex.com

Accepted for publication 11 March 2004

\section{REFERENCES}

1 Edoute $\mathrm{Y}$, Farraf $\mathrm{N}$, Kerner $\mathrm{H}$, et al. Myelofibrosis with bleeding jejunoileal angiodysplasia diagnosed by intraoperative endoscopy. Postgrad Med J 1991:67:859-60.

2 Freedman SD, Drews RE, Glotzer DJ, et al. Recurrent gastrointestinal bleeding associated with myelofibrosis and diffuse intestinal telangiectasia. Gastroenterology 1991;101:1432-9.

3 Foutch PG. Colonic angiodysplasia. Gastroenterologist 1997;5:148-56.

4 Naveau S, Bedossa P, Poynard T, et al. Portal hypertensive colopathy. A new entity. Dig Dis Sci 1991;36:1774-81.

5 Jacobs P, Maze S, Tayob F, et al. Myelofibrosis, splenomegaly, and portal hypertension. Acta Haematol 1985;74:45-8.

6 Balzer C, Lotterer E, Kleber G, et al. Transjugular intrahepatic portosystemic shunt for bleeding and angiodysplasia-like lesions in portal-hypertensive colopathy. Gastroenterology 1998;115:167-72.

7 Kouroumalis EA, Koutroubakis IE, Manousos ON. Somatostatin for acute severe bleeding from portal hypertensive gastropathy. Eur J Gastroenterol Hepatol 1998; 10:509-12. 